\title{
Pulmonary venous isolation by antral ablation with a large cryoballoon for treatment of paroxysmal and persistent atrial fibrillation: medium-term outcomes and non-randomised comparison with pulmonary venous isolation by radiofrequency ablation
}

\author{
Pipin Kojodjojo, Mark D O’Neill, Phang Boon Lim, Louisa Malcolm-Lawes, \\ Zachary I Whinnett, Tushar V Salukhe, Nicholas W Linton, David Lefroy, \\ Anthony Mason, Ian Wright, Nicholas S Peters, Prapa Kanagaratnam, D Wyn Davies
}

Department of Cardiac Electrophysiology, St. Mary's Hospital, Imperial College NHS Healthcare Trust, London, UK

\section{Correspondence to} Pipin Kojodjojo, St. Mary's Hospital, Imperial College Healthcare NHS Trust, Praed Street, London W2 1NY, United Kingdom;

pipin.kojodjojo@imperial.ac.uk

Accepted 4 May 2010

\begin{abstract}
Background To prevent atrial fibrillation (AF) recurrence after catheter ablation, pulmonary venous isolation (PVI) at an antral level is more effective than segmental ostial ablation. Cryoablation around the pulmonary venous (PV) ostia for AF therapy is potentially safer compared to radiofrequency ablation (RFA). The aim of this study was to investigate the efficacy of a strategy using a large cryoablation balloon to perform antral cryoablation with 'touch-up' ostial cryoablation for PVI in patients with paroxysmal and persistent AF.

Methods Paroxysmal and persistent AF patients
\end{abstract} undergoing their first left atrial ablation were recruited. After cryoballoon therapy, each PV was assessed for isolation and if necessary, treated with focal ostial cryoablation until PVI was achieved. Follow-up with Holter monitoring was performed. Clinical outcomes of the cryoablation protocol were compared, with consecutive patients undergoing PVI by RFA.

Results 124 consecutive patients underwent cryoablation. $77 \%$ of paroxysmal and $48 \%$ of persistent AF subjects were free from AF at 12 months after a single procedure. Over the same time period, 53 consecutive paroxysmal AF subjects underwent PVI with RFA and at 12 months, $72 \%$ were free from AF at 12 months $(p=N S)$. There were too few persistent AF subjects $(n=8)$ undergoing solely PVI by RFA as a comparison group. Procedural and fluoroscopic times during cryoablation were significantly shorter than RFA. Conclusions PV isolation can be achieved in less than $2 \mathrm{~h}$ by a simple cryoablation protocol with excellent results after a single intervention, particularly for paroxysmal AF.

\section{BACKGROUND}

Pulmonary venous isolation (PVI) remains the cornerstone of most atrial fibrillation (AF) ablation procedures. On its own, it is successful in preventing recurrence of paroxysmal AF in $60-80 \%$ of subjects undergoing PVI after a single procedure. ${ }^{12}$ In ablation of persistent AF, PVI is usually the first step in a stepwise approach to terminate $\mathrm{AF}^{3}$

The shallow learning curve in performing PVI is partly due to variable PV anatomy, which results in difficulty in appreciating the complex three-dimensional anatomy of the left atrium (LA) and the pulmonary veins and in catheter manipulation around the LA and PV ostia. ${ }^{4}$ Pulmonary venous isolation at the antral level is often preferred in order to include ostial foci within the level of isolation and minimise the risk of PV stenosis. To this extent, antral ablation has been shown to be more effective than ostial segmental ablation in preventing AF recurrence. ${ }^{5}$

With these considerations in mind, ablation technology that could deliver circumferential antral ablation centred on the PV ostia would facilitate AF ablation. Although a range of different ablation energy sources (most commonly radiofrequency energy and cryotherapy) are available, cryotherapy offers a theoretical advantage in that it does not disrupt target tissue architecture and could reduce complications such as pulmonary venous stenosis and atrio-oesphageal fistulation. ${ }^{67}$ To date, no case of pulmonary venous stenosis, atrio-oesophageal fistulation or coronary occlusion has been reported during cryoablation for AF. In this study, results are reported of a protocol incorporating the use of a large cryoablation balloon to perform antral cryoablation with 'touch-up' focal ostial cryoablation to isolate PVs in patients with paroxysmal and persistent AF undergoing their index ablation procedure. In addition, the medium-term results of the large cryoablation balloon protocol were compared with those from consecutive AF patients undergoing PVI using conventional radiofrequency ablation. (RFA)

\section{METHODS}

\section{Patient selection}

Patients with symptomatic, medically refractory AF referred for ablation from May 2006 to May 2009 were recruited. Subjects had either paroxysmal AF (defined as self-terminating AF episodes lasting $<7$ days) or early persistent AF (defined as episodes of $\mathrm{AF}$ that lasted $>7$ days, requiring direct current cardioversion to restore sinus rhythm and transition from a clinical pattern of paroxysmal AF in the past 12 months). All patients were undergoing their first AF ablation procedure.

Informed consent was obtained from all patients prior to the procedure. All antiarrhythmic agents, 
except amiodarone, were stopped at least 5 half lives before the procedure. In patients receiving oral anticoagulants, warfarin was stopped 5 days prior to the procedure with bridging tinzaparin $(175 \mathrm{IU} / \mathrm{kg})$. On the day of the procedure, transoesophageal echocardiography excluded intracardiac thrombus. No preprocedural imaging was performed to assess pulmonary venous anatomy.

\section{Cryoablation protocol}

Venous access was obtained from the right femoral vein, through which two 8 French sheaths (SR0 sheath, St. Jude Medical, St Paul, Minnesota, USA) were introduced into the left atrium via two separate transeptal punctures. A curvilinear mapping catheter (Inquiry Optima, St Jude Medical, USA or Lasso, Biosense Webster, Diamond Bar, California, USA) was introduced via one of the transeptal sheaths to map the PV ostia. Contrast pulmonary venography was performed with a 7 French $\mathrm{NIH}$ catheter for all targeted PVs and the PV mapping catheter was positioned at every targeted PV ostium to confirm electrical connection of the PV at baseline. Over an exchange length 0.035 inch guidewire introduced into the left superior $\mathrm{PV}$, an $8 \mathrm{Fr}$ transeptal sheath was exchanged for a 15 Fr deflectable sheath (Flexcath, Medtronic, Minneapolis, Minnesota, USA) to allow introduction of a $28 \mathrm{~mm}$ cryoablation balloon (Arctic Front, Medtronic, USA). This was the only cryoballoon used for all cases. The Arctic Front cryoballoon is a deflectable 'balloon within a balloon' catheter, whereby refrigerant is delivered into the inner balloon. A constant vacuum applied between the inner and outer balloon prevents the leakage of refrigerant into the circulation in the event of a defect in the inner balloon. Over a guidewire introduced fluoroscopically into each targeted PV, the cryoballoon was inflated in the left atrium before it was advanced into the PV antrum (figure 1). Any point of contact between the endothelium and balloon was ablated. A deflectable quadripolar catheter was introduced via the left femoral vein for either differential atrial pacing or pacing in the superior vena cava to capture the right phrenic nerve.
After the introduction of the Arctic Front catheter into each vein sequentially over a 0.035 -inch guidewire, $50 \%$ contrast was injected distally via the central lumen to confirm a good seal between the balloon and the pulmonary venous antra. Two 5 min cryoballoon applications were applied to each $\mathrm{PV}$, aiming for a trough temperature of less than $-40^{\circ} \mathrm{C}$. If a good PV seal could not be achieved, the orientation of the Arctic Front catheter was adjusted by either deflecting the catheter and/or Flexcath guidewire or by manipulating the guidewire into a different branch of the PV. The lowest (trough) temperature achieved during each cryoballoon application was recorded.

During cryoballoon applications to the right-sided PVs, the right phrenic nerve was captured by pacing (1500 ms CL, $20 \mathrm{~mA}$ output) in the superior vena cava. The operator monitored the strength of right hemidiaphragmatic contractions by palpation in the right hypochondrium to quickly detect injury to the right phrenic nerve. Cryoballoon applications were immediately terminated when any attenuation of the strength of right hemidiaphragmatic contractions was felt.

After each PV antrum was treated with two cryoballoon applications, the PV ostia were remapped with the curvilinear mapping catheter and any residual LA-PV connections were treated by focal cryoablation (Freezor Max, Medtronic, USA) to achieve PV isolation. This electrophysiological end point of entrance block was reconfirmed at the end of the procedure. In persistent AF patients, direct current cardioversion was performed to restore sinus rhythm. If typical atrial flutter had been previously documented during electrocardiographic monitoring, cavotricuspid isthmus ablation was performed using an $8 \mathrm{~mm}$ tipped radiofrequency catheter (IBI, St Jude Medical, USA) (60W, $60^{\circ} \mathrm{C}$ ) until bidirectional isthmus block was achieved.

\section{Pulmonary venous isolation by conventional radiofrequency ablation}

Consecutive patients undergoing PVI using conventional radiofrequency ablation at St Mary's Hospital, London, from May
Figure 1 Positions of inflated $28 \mathrm{~mm}$ cryoballoon at the four pulmonary venous antra in the same paroxysmal atrial fibrillation (AF) patient. Note the hold-up of contrast in the pulmonary veins due to balloon occlusion of the antra. A quadripolar catheter is positioned in the superior vena cava to capture the right phrenic nerve. All four pulmonary veins (PV) were isolated after two cryoballoon applications to each PV.
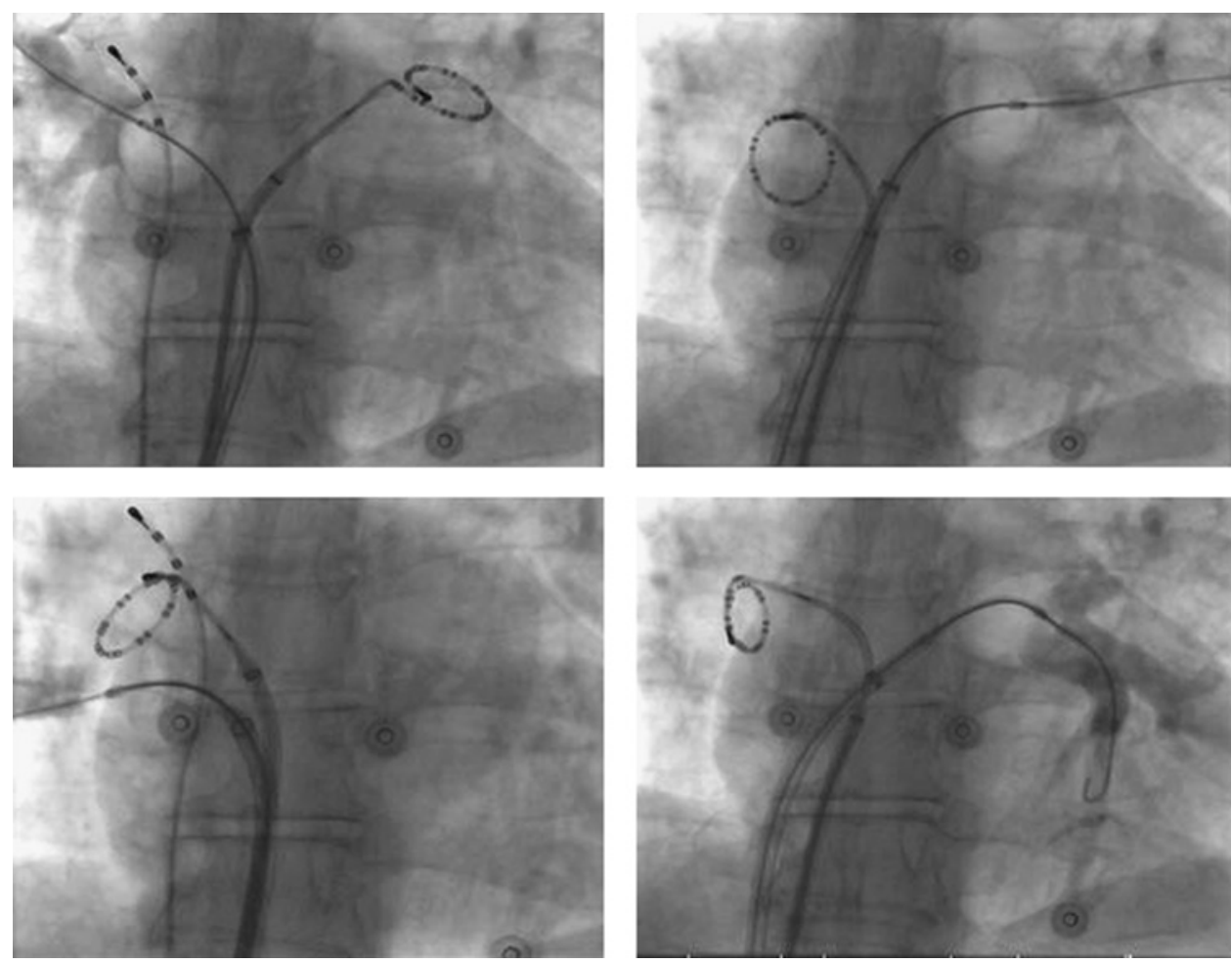
2006 to May 2009 were recruited as a comparison group. The following groups of patients were excluded:

A. those undergoing repeat procedures for recurrent $\mathrm{AF}(\mathrm{n}=90)$;

B. those undergoing pulmonary venous isolation using other investigational devices such as the Hansen Robotic Catheter system (Hansen Medical, Mountain View, California, USA), MeshAblator (Bard Inc., Lowell, Massachusetts, USA), Ablation Frontiers (Medtronic, USA), etc. $(n=108)$;

C. those who received additional left atrial ablation lesions such as ablation of complex fractionated atrial electrograms or linear lesions $(\mathrm{n}=87)$.

Circumferential antral ablation using a $4 \mathrm{~mm}$ cooled tipped catheter (Thermocool, Biosense Webster, USA), limited to $25-35 \mathrm{~W}$ and $17 \mathrm{ml} / \mathrm{min}$ flow was performed around ipsilateral pairs of pulmonary veins, guided by either fluoroscopy or a three-dimensional mapping system (Carto, Biosense Webster, USA). Following the creation of two encircling lesion sets, additional ostial segmental ablation guided by a curvilinear mapping catheter was carried out until veins were isolated with entrance block set as the same electrophysiological end point. Similarly, if typical atrial flutter had been documented during electrocardiographic monitoring either before or during the procedure, cavotricuspid isthmus ablation was performed using the same catheter $(35 \mathrm{~W}, 17 \mathrm{ml} / \mathrm{min})$, until bidirectional isthmus block was achieved.

\section{Postoperative care and follow-up}

After the procedure, oral anticoagulation with warfarin was resumed with bridging tinzaparin continued until international normalised ratios (INRs) were therapeutic. All antiarrhythmic agents that were discontinued prior to the procedure were recommenced, with a view to stopping all antiarrhythmic agents after 3 months if patients remained free from AF. A 3-month blanking period was applied such that any AF recurrence during this period was not considered as treatment failure. Follow-up was performed by clinic visits at 1, 3, 6 and subsequently 6 monthly intervals with repeated $24 \mathrm{~h}$ Holter monitoring and event recorders. All patients had at least 6 months follow-up. Recurrence was defined as any documented episode of AF (both symptomatic and asymptomatic) or atrial tachycardia lasting for more than $30 \mathrm{~s}$.

\section{Statistical analysis}

Continuous data are given as mean \pm standard deviation. Student $t$ test was used to compare differences between groups. Recurrence-free survival over time was calculated by Kaplan-Meier method and $p<0.05$ was considered significant. All analyses were performed using Prism 4.0. (Graphpad, USA)

\section{RESULTS}

\section{Patient characteristics}

In total, 177 patients were recruited in a 3-year period from May 2006 to May 2009. Of these, 124 patients underwent cryoablation. The mean age of patients undergoing cryoablation was $58.5 \pm 9.8$ years, with $77 \%$ being men. Nearly all subjects had normal left ventricular function. Hypertension was the most common comorbidity. Ten per cent of subjects had previously undergone cavotricuspid isthmus ablation for typical atrial flutter. The persistent AF group was older and had larger left atria compared to the paroxysmal AF group. Baseline characteristics of paroxysmal and persistent AF patients undergoing cryoablation are shown in table 1.

Over the same period, 53 subjects with paroxysmal AF underwent PVI using RFA. Only eight persistent AF subjects
Table 1 Baseline characteristics of paroxysmal and persistent atrial fibrillation (AF) subjects undergoing cryoablation

\begin{tabular}{llll}
\hline & $\begin{array}{l}\text { Paroxysmal } \\
\mathbf{A F}(\mathbf{n = 9 0})\end{array}$ & $\begin{array}{l}\text { Persistent } \\
\mathbf{A F}(\mathbf{n}=\mathbf{3 4})\end{array}$ & $\mathbf{p}$ \\
\hline Age (years) & $57.3 \pm 9.4$ & $61.7 \pm 10.4$ & 0.01 \\
Men (\%) & 75 & 82 & NS \\
Mean LA diameter (mm) & $39.6 \pm 7.1$ & $43.1 \pm 7.0$ & 0.04 \\
LVEDD (mm) & $49.5 \pm 6.7$ & $50.9 \pm 7.4$ & NS \\
Ejection fraction (\%) & $65.0 \pm 8.8$ & $64.0 \pm 10.1$ & NS \\
$\begin{array}{l}\text { Duration of AF (years) } \\
\text { Number of failed antiarrhythmic drugs }\end{array}$ & $5.6 \pm 4.1$ & $5.8 \pm 3.3$ & NS \\
Comorbidities & $2.1 \pm 0.6$ & $2.1 \pm 0.6$ & NS \\
Hypertension & $42(47 \%)$ & $20(59 \%)$ & \\
- Coronary artery disease requiring & $5(6 \%)$ & 0 & \\
$\quad$ CABG or PCl & & & \\
Prior ablations & & & \\
Cavotricuspid isthmus ablation & $10(11 \%)$ & $3(9 \%)$ & \\
\hline CABG, coronary artery bypass graft; LA, left atrium; LVEDD, left ventricular end diastolic
\end{tabular}
diameter; $\mathrm{PCl}$, percutaneous coronary intervention.

had solely PVI as their index procedure, and this small cohort size precluded any meaningful comparison of outcomes between RFA and cryoablation of persistent AF. There were no significant differences in baseline characteristics between paroxysmal AF subjects undergoing either cryoablation or RFA (table 2).

\section{Acute procedural results Cryoablation}

Four-hundred and ninety-two PVs were targeted. Four right inferior pulmonary veins were too small to introduce the PV mapping catheter to confirm isolation. Mean procedural and fluoroscopic times were $110 \pm 27$ and $28 \pm 10 \mathrm{~min}$ respectively. Although $83 \%$ of pulmonary veins were isolated with cryoballoon applications alone, only $40 \%$ of patients had all targeted PVs isolated with the use of the cryoballoon alone with two $300 \mathrm{~s}$ applications to each PV. Ostial focal cryoablation to a mean of 1.5 PV was required in the remaining $60 \%$ of patients. In all $\mathrm{PVs}$ except the left upper pulmonary vein (LUPV), PV isolation was associated with lower mean trough temperature achieved during balloon cryotherapy (table 3). All but two persistent AF subjects required cardioversion at the end of procedure to restore sinus rhythm. In the two subjects, AF organised into typical atrial flutter, which terminated into sinus rhythm during cavotricuspid isthmus ablation. After left atrial cryotherapy, 17 paroxysmal AF

Table 2 Comparison between paroxysmal atrial fibrillation (AF) subjects undergoing cryoablation and radiofrequency ablation

\begin{tabular}{llll}
\hline Paroxysmal AF subjects undergoing & $\begin{array}{l}\text { Cryoablation } \\
(\mathbf{n = 9 0 )}\end{array}$ & $\begin{array}{l}\text { Conventional } \\
\text { Radiofrequency } \\
\text { Ablation }(\mathbf{n = 5 3 )}\end{array}$ & $\mathbf{p}$ \\
\hline Age (years) & $57.3 \pm 9.4$ & $59.3 \pm 9.7$ & NS \\
Men (\%) & 75 & 77 & NS \\
Mean LA diameter (mm) & $39.6 \pm 7.1$ & $41.6 \pm 6.5$ & NS \\
LVEDD (mm) & $49.5 \pm 6.7$ & $47.8 \pm 6.5$ & NS \\
Ejection fraction (\%) & $65.0 \pm 8.8$ & $60.3 \pm 7.3$ & NS \\
$\begin{array}{l}\text { Duration of AF (years) } \\
\text { Number of failed antiarrhythmic drugs }\end{array}$ & $5.6 \pm 4.1$ & $6.0 \pm 4.8$ & NS \\
Comorbidities & $2.1 \pm 0.6$ & $1.9 \pm 0.6$ & NS \\
- Hypertension & $42(47 \%)$ & $14(26 \%)$ & \\
- Coronary artery disease requiring & $5(6 \%)$ & $3(6 \%)$ & \\
$\quad$ CABG or PCl & & & \\
$\begin{array}{l}\text { Prior ablations } \\
\text { Cavotricuspid isthmus ablation }\end{array}$ & $10(11 \%)$ & $4(8 \%)$ & \\
\hline CABG, coronary artery bypass graft; LA, left atrium; LVEDD, left ventricular end diastolic \\
diameter; PCl, percutaneous coronary intervention. & &
\end{tabular}


Table 3 Acute success of $28 \mathrm{~mm}$ cryoballoon in achieving pulmonary venous isolation

\begin{tabular}{|c|c|c|c|c|}
\hline $\begin{array}{l}\text { Targeted } \\
\text { veins }\end{array}$ & $\begin{array}{l}\text { Isolated with } \\
\text { cryoballoon alone }\end{array}$ & $\begin{array}{l}\text { Mean trough } \\
\text { temperature } \\
\text { when isolation } \\
\text { achieved }\left({ }^{\circ} \mathrm{C}\right)\end{array}$ & $\begin{array}{l}\text { Mean trough } \\
\text { temperature } \\
\text { when isolation not } \\
\text { achieved }\left({ }^{\circ} \mathrm{C}\right)\end{array}$ & $\mathbf{p}$ \\
\hline LUPV & $83 \%$ & $-49.4 \pm 6.5$ & $-46.0 \pm 4.7$ & NS \\
\hline LLPV & $95 \%$ & $-45.5 \pm 7.5$ & $-40.7 \pm 4.9$ & $<0.01$ \\
\hline RUPV & $85 \%$ & $-49.1 \pm 5.4$ & $-45.6 \pm 7.9$ & 0.03 \\
\hline RLPV & $68 \%$ & $-42.1 \pm 6.9$ & $-38.4 \pm 4.5$ & $<0.01$ \\
\hline All PV & $83 \%$ & $-46.6 \pm 7.3$ & $-41.0 \pm 7.2$ & 0.02 \\
\hline All Patients & $40 \%$ & & & \\
\hline
\end{tabular}

LLPV, left lower pulmonary vein; LUPV, left upper pulmonary vein; RLPV, right lower pulmonary vein; RUPV, right upper pulmonary vein.

subjects and four persistent AF subjects had cavotricuspid isthmus ablation.

\section{Radiofrequency ablation}

In 53 paroxysmal AF subjects, 211 PVs were targeted and 99\% (209/211) of PVs were isolated. Six patients had cavotricuspid isthmus ablation after PVI. An electroanatomical mapping system was used in 51 out of 53 cases. Mean procedural and fluoroscopic times were $208 \pm 58$ and $62 \pm 36$ min respectively, which were significantly longer than cryoablation procedures for paroxysmal AF ( $p<0.001)$ (table 4).

\section{Follow-up}

Cryoablation

Mean follow-up was 13.3 \pm 7.4 months, with 644 Holter recordings analysed. Arrhythmia-free survival curves for the entire group, paroxysmal and persistent AF subjects are shown in figure 2. At 12 months follow-up, 77\% of the paroxysmal AF subjects and $48 \%$ of persistent AF subjects were free from AF recurrence after a single procedure. There was a significant difference in arrhythmia-free survival between the paroxysmal and persistent AF groups $(\mathrm{p}=0.002)$. Among patients free from $\mathrm{AF}$ at 12 months, nine out of 69 paroxysmal AF and two out of 15 persistent AF subjects were still on antiarrhythmic medication for frequent atrial ectopy. No organised atrial tachycardias were detected during follow-up, with AF being the only recurrent arrhythmia.

\section{Radiofrequency ablation}

At 12 months, $72 \%$ of paroxysmal AF subjects who underwent RFA (five on previously ineffective antiarrhythmics, 27 off antiarrhythmic agents) were free from AF compared to $77 \%$ of the paroxysmal AF subjects who underwent cryoablation (nine

Table 4 Comparison between paroxysmal atrial fibrillation (AF) subjects undergoing cryoablation and conventional radiofrequency ablation

\begin{tabular}{|c|c|c|c|}
\hline $\begin{array}{l}\text { Paroxysmal AF } \\
\text { subjects undergoing }\end{array}$ & $\begin{array}{l}\text { Cryoablation } \\
(n=90)\end{array}$ & $\begin{array}{l}\text { Conventional } \\
\text { radiofrequency } \\
\text { ablation }(n=53)\end{array}$ & p \\
\hline Follow-up (months) & $14.9 \pm 7.7$ & $15.6 \pm 7.4$ & NS \\
\hline $\begin{array}{l}\text { Additional } \\
\text { cavotricuspid ablation }\end{array}$ & 17 (19\%) & $6(11 \%)$ & \\
\hline Procedural time (min) & $108 \pm 28$ & $208 \pm 58$ & $<0.001$ \\
\hline Fluoroscopic time (min) & $27 \pm 9$ & $62 \pm 36$ & $<0.001$ \\
\hline Complications & $\begin{array}{l}\text { One pericardial } \\
\text { effusion, two transient } \\
\text { phrenic nerve palsies }\end{array}$ & $\begin{array}{l}\text { Two pericardial } \\
\text { effusion unrelated to } \\
\text { transeptal puncture } \\
\text { requiring drainage }\end{array}$ & \\
\hline
\end{tabular}

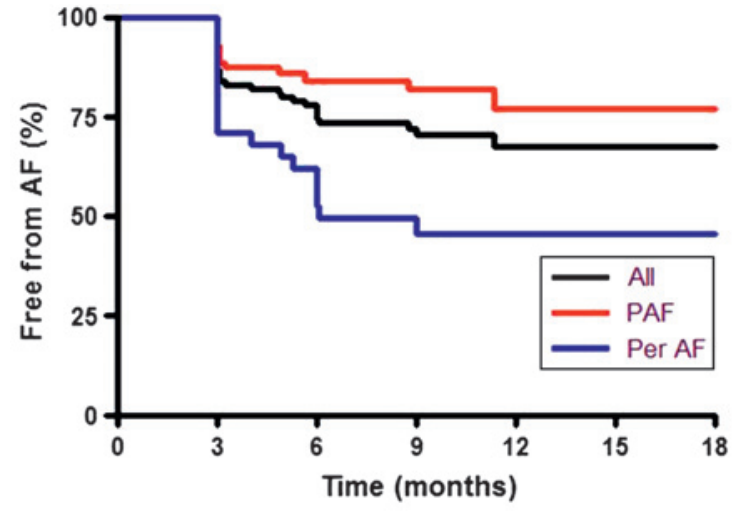

\begin{tabular}{lllll}
\hline Months after cryoablation & 0 & 6 & 12 & 18 \\
\hline All & 124 & 99 & 83 & 29 \\
\hline Paroxysmal AF & 90 & 79 & 69 & 26 \\
\hline Persistent AF & 34 & 21 & 15 & 4 \\
\hline
\end{tabular}

Figure 2 Survival curves of subjects undergoing cryoablation. PAF, paroxysmal atrial fibrillation; Per $\mathrm{AF}$, persistent atrial fibrillation. Comparison between PAF and Per AF curves, $\mathrm{p}=0.002$.

on previously ineffective antiarrhythmics, 60 off antiarrhythmics). Survival curves are shown in figure 3 , with no statistical difference between the two curves.

\section{Complications}

Five major complications occurred (table 4). Pericardial effusions requiring drainage occurred in two patients undergoing RFA and one patient undergoing cryoablation, in the latter due to a guidewire perforating the side-branch of the LUPV before any cryoablation was delivered. Uniquely in the cryoablation group, there were two cases of transient right phrenic nerve palsies, which resolved within 3 and 14 months. The phrenic nerve palsy that took 14 months to recover was caused by unmonitored cryoablation of the right lower PV. As a result of this patient, phrenic nerve monitoring was extended to include cryotherapy of the right lower PV, whereas earlier in the series it had only been applied to cryotherapy of the right upper PV.

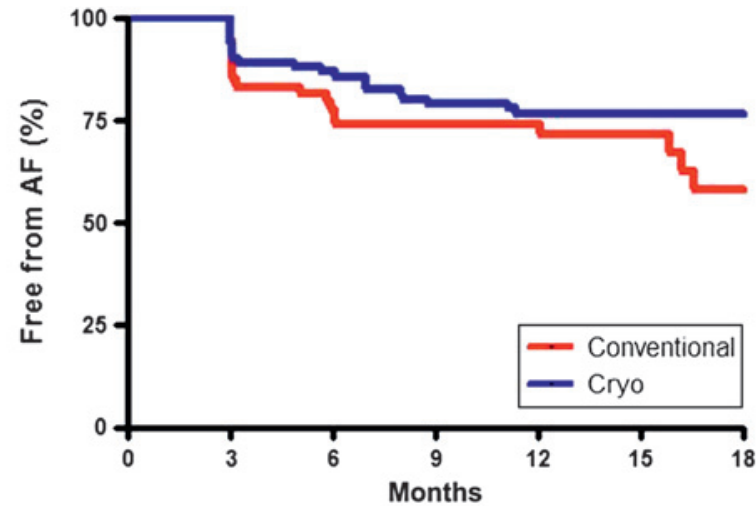

\begin{tabular}{lllll}
\hline Months after ablation & 0 & 6 & 12 & 18 \\
\hline Cryoablation & 90 & 79 & 69 & 26 \\
\hline Radiofrequency Ablation & 53 & 42 & 33 & 12 \\
\hline
\end{tabular}

Figure 3 Survival curves of paroxysmal atrial fibrillation subjects undergoing cryoablation and conventional radiofrequency ablation. Comparison between conventional radiofrequency ablation and cryoablation survival curves, $\mathrm{p}=\mathrm{NS}$. 


\section{Repeat ablation Cryoablation}

During follow-up, 17 (14\%) cryoablation patients had repeat ablation for recurrent AF. During their second procedure, 65 pulmonary veins targeted by cryoballoon ablation during the first procedure were re-studied. Twenty-seven per cent of PVs remained isolated. Forty-eight PVs demonstrated electrical reconnection (18 LUPV, 15 LLPV, five RUPV, and 10 RLPV). Seventy-four per cent of PVs that demonstrated reconnection were previously isolated with the cryoballoon alone, whereas $82 \%$ of PVs that maintained electrical isolation were previously isolated with the cryoballoon alone. The mean trough temperature reached during previous cryoballoon therapy to reconnected veins was higher compared to the temperature reached during previous cryoballoon therapy to persistently isolated veins $\left(-41.9 \pm 5.3^{\circ} \mathrm{C}\right.$ versus $\left.-45.2 \pm 4.6 \mathrm{C}, \mathrm{p}=0.03\right)$.

\section{Radiofrequency ablation}

During follow-up, 12 (23\%) patients who underwent PVI by RFA had a second procedure for recurrent AF. Out of $47 \mathrm{PVs}$ targeted and isolated during the first procedure, 11 PVs (23\%) remained isolated.

All reconnected PVs were re-isolated with RFA.

\section{DISCUSSION}

The present study is the first to show that a novel, empirical two freeze per vein strategy utilising a large cryoballoon to perform antral cryoablation followed by focal ostial cryoablation to isolate selected pulmonary veins results in medium-term outcomes comparable to RFA; with significantly reduced procedural and fluoroscopic times.

Although the benefits of cryoablation, such as minimal risks of PV stenosis, have long been recognised, an efficient delivery platform to deliver contiguous lesions around PV ostia has been lacking until the introduction of the Arctic Front catheter system. Pulmonary venous isolation using earlier delivery platforms such as $4 \mathrm{~mm}, 6 \mathrm{~mm}$ tipped and expandable circular cryoablation catheters required lengthy procedures of nearly $6 \mathrm{~h}$, and was associated with unsatisfactory clinical outcomes. ${ }^{8-11}$ This was thought to be due to the effects of competitive warming by blood flowing past the cryocatheter reducing lesion size. The Arctic Front catheter obstructs blood flow from the PV being treated, thereby removing this limitation.

Several centres have already reported on the feasibility of the cryoballoon to isolate PV for treatment of paroxysmal and persistent atrial fibrillation. ${ }^{12-16}$ In the largest series reported to date, Neumann et al investigated the efficacy of balloon cryoablation in 293 paroxysmal and 53 persistent AF subjects. ${ }^{15}$ Using a subjective grading of 1 to 4 to assess the degree of balloon occlusion during contrast venography, a complete occlusion was associated with an increased probability of PVI with the cryoballoon alone. After a median of 12 months follow-up, 74\% of paroxysmal and $42 \%$ of persistent AF patients were free from AF. Van Belle et al reported a $73 \%$ freedom from recurrent AF, using a 3-month blanking period. ${ }^{17}$ These results are consistent with the present findings. In addition, the present study has shown that a lower trough temperature during cryoballoon application is also associated with increased likelihood of acute isolation and maintenance of long-lasting electrical disconnection when restudied in patients undergoing repeat ablation procedures.

Some centres have used either preprocedural computer tomography (CT), magnetic resonance imaging or periprocedural fluoroscopy to evaluate the size of the pulmonary venous ostium before choosing to use either a smaller (23 $\mathrm{mm}$ ) or large (28 $\mathrm{mm}$ ) cryoballoon. ${ }^{13-15}$ It was believed that a closer match between the cryoballoon size and the size of PV ostium would allow for better balloon occlusion, and in turn result in more effective lesions. There were two potential disadvantages to this approach: first, the need for preprocedural CT imaging increased the cumulative radiation dose received by the patients and overall costs, and second, retrospective analysis suggests that the use of a smaller cryoballoon was associated with a higher risk of right phrenic nerve palsy through more distal right PV cryoablation. ${ }^{15}$

In two separate small series, detailed electroanatomical mapping was used before and after cryoballoon applications to determine the anatomical level at which PVI isolation was achieved. When the $23 \mathrm{~mm}$ balloon was used, the veins were found to be isolated at the level of the ostium, whereas use of the $28 \mathrm{~mm}$ larger balloon results in the formation of much wider, circumferential and antrally located lesions. ${ }^{18} 19$ In cadaveric studies, the right phrenic nerve is not uncommonly less than $2 \mathrm{~mm}$ away from the anterior aspect of the proximal segment of the right superior pulmonary vein. ${ }^{20}$ Therefore, it would be expected that distal ablation to the right-sided PV (which is more likely with a $23 \mathrm{~mm}$ balloon) will result in phrenic nerve injury. This complication appears to be unique to balloon-based catheter systems delivering cryoenergy, laser or focused ultrasound energy. ${ }^{21}$

Chun et al described the feasibility of using only the larger cryoballoon without preprocedural left atrial imaging in 27 paroxysmal AF subjects with the aim of achieving PV isolation at the antral level and minimising the risk of phrenic nerve palsy. ${ }^{12}$ Although isolation was achieved in $98 \%$ of targeted veins, three cases $(11 \%)$ of transient phrenic nerve palsy still occurred due to distal PV ablations. Unlike the present protocol, a larger number of cryoballoon applications were made particularly for the right inferior PV where a median of up to five $300 \mathrm{~s}$ applications were made. In that study, phrenic nerve dysfunction was defined as the loss of reliable capture from pacing in the superior vena cava. The lower rate of phrenic nerve palsy in the present study (1.6\%), the lowest amongst all published series, is most likely due to a combination of limiting cryoballoon applications to two per vein, pacing the right phrenic nerve at a higher frequency of 40 contractions/min and by palpating for a reduction in the strength of diaphragmatic contractions in the right hypochondria, which in the authors' experience, precedes the onset of complete phrenic nerve palsy. The phrenic nerve palsy that took 14 months to recover was caused by unmonitored cryoablation of the right lower PV. As a result of this patient, phrenic nerve monitoring was extended to include cryotherapy of the right lower PV, whereas, earlier in the series, it had only been applied to cryotherapy of the right upper PV.

The clinical outcomes in the present study are in agreement with previous studies for patients with paroxysmal AF and structurally normal hearts; that PV isolation as the sole procedure is effective in preventing AF recurrence in the majority of patients. However, in subjects with persistent AF, additive ablation strategies, combined with PVI, are required to maintain long-term sinus rhythm. The present definition of persistent AF was not consistent with those defined in international guidelines, but was chosen arbitrarily with the expectation that patients with a more recent transition from a clinical pattern of paroxysmal AF to persistent AF would exhibit a lesser degree of substrate remodelling and respond more favourably to PV isolation alone. Disappointingly, the medium-term clinical outcome for the persistent AF group was significantly lower than paroxysmal AF group and it is likely that additional ablation lesions will be required during their index procedure to achieve higher first-procedural success rates. 


\section{Limitations}

This report details clinical results of a novel cryoablation protocol for AF therapy. The comparison between RFA and cryoballoon was not randomised, although consecutive patients undergoing PVI by different operators in the same institution in a real-life clinical setting were recruited. The decision to apply only two cryoballoon applications per vein was based on early experience with the Arctic Front and ultrasound balloon catheters; and in keeping with other studies whereby a median of two applications was required to isolate all PVs except the right inferior PV. Only $24 \mathrm{~h}$ Holter monitoring was performed to detect asymptomatic AF and more episodes may have been detected if longer recording durations were used. Limiting the total number of cryoballoon applications allowed for a shorter procedural time but did increase procedural cost, as $60 \%$ of the cryoablation cohort required a second catheter to ensure achievement of PV isolation.

\section{CONCLUSIONS}

Pulmonary venous isolation can be achieved in less than $2 \mathrm{~h}$ by a simple cryoablation protocol with excellent results after a single intervention, particularly for paroxysmal AF. Monitoring of phrenic nerve function by continuous pacing and palpating the vigour of diaphragmatic contractions is essential in minimising the risk of phrenic nerve palsy during balloon cryoablation.

Funding British Heart Foundation. Dr Pipin Kojodjojo is funded by a British Heart Foundation Travel Fellowship (FS/09/047).

Competing interests None.

Ethics approval This study was conducted with the approval of St. Mary's Hospital, Imperial College Healthcare NHS Trust.

Provenance and peer review Not commissioned; externally peer reviewed.

\section{REFERENCES}

1. Bhargava M, Di Biase L, Mohanty P, et al. Impact of type of atrial fibrillation and repeat catheter ablation on long-term freedom from atrial fibrillation: Results from a multicenter study. Heart Rhythm 2009;6:1403-12.

2. Jais $\mathbf{P}$, Hocini $\mathbf{M}$, Sanders $\mathbf{P}$, et al. Long-term evaluation of atrial fibrillation ablation guided by noninducibility. Heart Rhythm 2006;3:140-5.

3. O'Neill MD, Wright M, Knecht S, et al. Long-term follow-up of persistent atrial fibrillation ablation using termination as a procedural endpoint. Eur Heart $J$ 2009;30:1105-12.
4. Marom EM, Herndon JE, Kim YH, et al. Variations in pulmonary venous drainage to the left atrium: implications for radiofrequency ablation. Radiology 2004;230:824-9.

5. Oral H, Scharf C, Chugh A, et al. Catheter ablation for paroxysmal atrial fibrillation: segmental pulmonary vein ostial ablation versus left atrial ablation. Circulation 2003;108:2355-60.

6. Khairy P, Dubuc M. Transcatheter cryoablation part I: preclinical experience. Pacing Clin Electrophysiol 2008;31:112-20.

7. Lemola K, Dubuc M, Khairy P. Transcatheter cryoablation part II: clinical utility. Pacing Clin Electrophysiol 2008;31:235-44.

8. Moreira W, Manusama R, Timmermans C, et al. Long-term follow-up after cryothermic ostial pulmonary vein isolation in paroxysmal atrial fibrillation. $J$ Am Coll Cardiol 2008;51:850-5.

9. Tse HF, Reek S, Timmermans $C$, et al. Pulmonary vein isolation using transvenous catheter cryoablation for treatment of atrial fibrillation without risk of pulmonary vein stenosis. J Am Coll Cardiol 2003;42:752-8.

10. Wong T, Markides V, Peters NS, et al. Percutaneous pulmonary vein cryoablation to treat atrial fibrillation. J Interv Card Electrophysiol 2004:11:117-26.

11. Wong T, Markides V, Peters NS, et al. Percutaneous isolation of multiple pulmonary veins using an expandable circular cryoablation catheter. Pacing Clin Electrophysiol 2004; 27:551-4.

12. Chun KR, Schmidt B, Metzner A, et al. The 'single big cryoballoon' technique for acute pulmonary vein isolation in patients with paroxysmal atrial fibrillation: a prospective observational single centre study. Eur Heart $J$ 2009;30:699-709.

13. Klein G, Oswald H, Gardiwal A, et al. Efficacy of pulmonary vein isolation by cryoballoon ablation in patients with paroxysmal atrial fibrillation. Heart Rhythm 2008;5:802-6.

14. Malmborg $\mathbf{H}$, Lonnerholm S, Blomstrom-Lundqvist C. Acute and clinical effects of cryoballoon pulmonary vein isolation in patients with symptomatic paroxysmal and persistent atrial fibrillation. Europace 2008;10:1277-80.

15. Neumann T, Vogt J, Schumacher B, et al. Circumferential pulmonary vein isolation with the cryoballoon technique results from a prospective 3-center study. J Am Coll Cardiol 2008;52:273-8.

16. Van Belle $\mathbf{Y}$, Janse $\mathrm{P}$, Rivero-Ayerza $\mathrm{MJ}$, et al. Pulmonary vein isolation using an occluding cryoballoon for circumferential ablation: feasibility, complications, and short-term outcome. Eur Heart J 2007;28:2231-7.

17. Van Belle $\mathbf{Y}$, Janse $P$, Theuns $D$, et al. One year follow-up after cryoballoon isolation of the pulmonary veins in patients with paroxysmal atrial fibrillation. Europace 2008:10:1271-6.

18. Reddy VY, Neuzil P, d'Avila A, et al. Balloon catheter ablation to treat paroxysmal atrial fibrillation: what is the level of pulmonary venous isolation? Heart Rhythm 2008:5:353-60.

19. Van Belle $\mathbf{Y}$, Knops $P$, Janse $P$, et al. Electro-anatomical mapping of the left atrium before and after cryothermal balloon isolation of the pulmonary veins. J Interv Card Electrophysiol 2009;25:59-65.

20. Sanchez-Quintana D, Cabrera JA, Climent V, et al. How close are the phrenic nerves to cardiac structures? Implications for cardiac interventionalists. J Cardiovasc Electrophysiol 2005;16:309-13.

21. Okumura $\mathbf{Y}$, Kolasa MW, Johnson SB, et al. Mechanism of tissue heating during high intensity focused ultrasound pulmonary vein isolation: implications for atrial fibrillation ablation efficacy and phrenic nerve protection. J Cardiovasc Electrophysiol 2008; 19:945-51. 